\title{
MICRODUREZA SUPERFICIAL DE MOLARES ARTIFICIALES DE RESINA ACRÍLICA UTILIZADOS EN ODONTOLOGÍA REHABILITADORA, TRES MARCAS COMERCIALES. ESTUDIO IN-VITRO.
}

\author{
SUPERFICIAL MICRO-STRENGTH OF ARTIFICIAL MILLS OF ACRYLIC RESIN USED IN \\ REHABILITATING DENTISTRY, THREE TRADE MARKS. IN-VITRO STUDIO
}

\author{
Paul Cruz Gallegos ${ }^{1 a}$, Alexander Cruz Gallegos ${ }^{2 a}$, Vinicio Santillán Cruz ${ }^{1 a b}$
}

\begin{abstract}
RESUMEN
Objetivo: El propósito del presente estudio fue determinar y comparar la microdureza superficial de tres marcas de molares artificiales para comprobar si existe diferencia significativa entre las mismas. Materiales y Métodos: Se utilizó molares artificiales a base de resina acrílica, 15 molares inferiores de cada marca TRILUX®, Gnathostar $\circledast$ y Duratone -n®. Se preparó las muestras realizando un corte transversal a nivel coronal para obtener una superficie plana, lisa y pulida. Se colocaron e $\mathrm{n}$ troqueles de tubo PVC con acrílico autopolimerizable de 1,5 cm. Se tinturó la superficie a analizar de color azul, para luego llevar al microdurómetro Metkon Duroline-M donde se realizó 3 indentaciones por cada muestra con una fuerza de 200 gramos durante 15 segundos, obteniendo los resultados en unidades de Vickers (Hv). Resultados: Los molares de la marca TRILUX® presentaron mayor microdureza superficial con un promedio de $24.306 \pm 1.41 \mathrm{Hv}$, seguidos de los morales Duratone-n® con $22.21 \pm 0.72 \mathrm{Hv}$ y, por último, Gnathostar ${ }^{\circledR}$ con $19.82 \pm 0.66 \mathrm{Hv}$. Mediante pruebas estadísticas se pudo confirmar que las tres medidas son totalmente diferentes, por lo cual podemos decir que existe diferencia significativa en cuanto a la microdureza superficial entre las tres marcas. Conclusiones: Los molares artificiales de la marca TRILUX® presentaron mayor microdureza superficial en comparación con los molares de resina acrílica Gnathostarß y Duratone -n®.
\end{abstract}

Palabras clave: arcada edéntula, diente artificial, pruebas de dureza. (Fuente: DeCS BIREME)

\begin{abstract}
Objectives: The purpose of the present study was to determine and compare the surface microhardness of three brands of artificial molars to verify if there is a significant difference between them. Materials and Methods: artificial molars were used based on acrylic resin, 15 lower molars of each brand TRILUX®, Gnathostar $\AA$ and Duratone-n®, the samples were prepared making a coronal cross section to obtain a flat, smooth surface and polished, it was placed in pvc dies with $1.5 \mathrm{~cm}$ Autopolymerizable acrylic, the surface to be analyzed was dyed blue, to then be taken to the Duroline-M Metkon Microdurometer where 3 indentations were made for each sample with a force of 200 grams during 15 seconds obtaining the results in units of Vickers (Hv). Results: The TRILUX® brand molars had higher surface microhardness with an average of $24,306 \pm 1.41 \mathrm{Hv}$, followed by the morals Duratone- $n \AA$ with $22.21 \pm 0.72 \mathrm{Hv}$ and finally Gnathostar® with $19.82 \pm 0.66 \mathrm{Hv}$, by statistical tests. He was able to confirm that the three measurements are totally different, which is why we can say that there is a significant difference in terms of surface microhardness between the three brands. Conclusions: The TRILUX® brand artificial molars had a higher superficial microhardness compared to the molars of Gnathostar $\AA^{\circledR}$ and Duratone-n® acrylic resin.
\end{abstract}

Key words: jaw, edentulous, tooth, artificial, hardness tests. (Source: MeSH NLM)

Recibido: 02 de enero 2018

Aprobado: 30 de mayo 2018

Publicado: 30 de junio 2018

${ }^{1}$ Universidad Central del Ecuador

Universidad Tecnológica Equinoccial.

${ }^{\mathrm{a} O d o n t o ́ l o g o ~-~}{ }^{\mathrm{b}}$ Docente

Correspondencia:

Alexander Cruz Gallegos.

Correo electrónico: alexandercruz1993@hotmail.com
Este es un artículo Open Access distribuido bajo la licencia Creative Commons Atribución-NoComercial- Compartir Igual 4.0

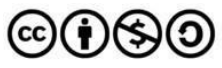

Citar como: Cruz Gallegos P., Cruz Gallegos A., Santillán Cruz V. Microdureza superficial de molares artificiales de resina acrílica utilizados en odontología rehabilitadora, tres marcas comerciales. Estudio in-vitro. KIRU. 2018 abr-jun; 15(2): 69-76 doi.org/10.24265/kiru.2018.v15n2.02 


\section{INTRODUCCIÓN}

La salud bucal asegura varios procesos, entre ellos la masticación, ayudando a la digestión de alimentos de manera adecuada. Por tal motivo, si existe un descuido en la misma puede afectar a todo el organismo ${ }^{(1)}$. Con el adelanto tecnológico en Odontología y las técnicas de prevención, aún es muy frecuente la pérdida de órganos dentarios, sobre todo con el aumento de población geriátrica, afectando primordialmente al sistema masticatorio y produciendo daños a nivel general ${ }^{(2)}$.

La principal molestia que se presenta en un paciente geriátrico es la pérdida de eficiencia masticatoria, ocasionada por la pérdida de piezas dentarias. Por ello, en el campo de la odontología se ve la importancia de rehabilitar a estos pacientes, no solo por estética, sino también por función y así establecer un equilibrio del sistema estomatognático ${ }^{(2)}$.

Minaya-Sánchez y cols. afirman que "de acuerdo con el tipo de diente, los molares son extraídos principalmente por caries y los dientes anteriores por enfermedad periodontal" (3), para lo cual la rehabilitación oral es una especialidad de la odontología encargada de la restauración; es decir, se encarga de devolver la función masticatoria, la estética y la armonía dental. Existen diferentes alternativas de tratamiento, como son las prótesis parciales y totales, en las cuales se usan dientes artificiales de resina acrílica.

Uno de los principales problemas que se presenta en los dientes de resina acrílica es el desgaste que estos presentan dentro de cavidad bucal. Los dientes posteriores son los que más desgaste presentan en relación con los del segmento anterior, provocando interferencias oclusales y disminución de la dimensión vertical del paciente, lo que causa mayor estrés en el proceso alveolar y puede provocar reabsorción del mismo. El desgaste de los dientes utilizados para la elaboración de prótesis está muy relacionado con la masticación, la fuerza oclusal, el tiempo de uso de las prótesis, hábitos dietéticos, entre otros ${ }^{(4)}$. Según la Asociación Dental Norteamericana (ADA), la Organización Internacional de Estandarización (ISO), la Academia de Prótesis Dentales (ahora denominada "Academy of Prosthodontics") y el Instituto Colombiano de Normas Técnicas y Certificación (Icontec), en relación con la dureza de los dientes artificiales, en estos se necesita que el diente pueda resistir las fuerzas a las que se somete durante la masticación y que durante el desgaste que presenten no liberen sustancias tóxicas al organismo ${ }^{(5)}$.

En el paciente endéntulo parcial o total, las funciones del sistema estomatognático se ven afectadas. Una de las alternativas es la rehabilitación con prótesis bucal, en la cual incluyen el uso de dientes artificiales. Estos deben tener similitud con la dentición natural en cuanto a sus propiedades para así brindar al paciente comodidad, estética y sobre todo devolver una oclusión adecuada, logrando así restablecer la función de sistema estomatognático ${ }^{(6)}$.

En la actualidad a pesar del gran impacto obtenido por la implantología en rehabilitación de pacientes desdentados, sean estos totales o parciales, aún existe un gran número de población que no goza del acceso a este tipo de tratamiento ya sea por el alto costo, anatomía, psicología o por problemas de salud en general, teniendo como opciones de tratamiento las prótesis totales o parciales removibles ${ }^{(7)}$.

Al hablar de salud bucodental, podemos decir que se trata no solo de tener una agradable sonrisa y dientes sanos, sino que la cavidad bucal es el espejo del cuerpo y en ella se puede ver reflejados algunas manifestaciones clínicas de enfermedades sistémicas. La salud bucodental y la salud general están íntimamente relacionadas y deben ser consideradas de manera integral ${ }^{(8)}$.

La pérdida de piezas dentarias desde el punto de vista estético puede ocasionar un gran impacto psicológico para el paciente, afectando más que la propia pérdida de función masticatoria. En la actualidad la pérdida de dientes, especialmente en el segmento anterior, se ve como un estigma social. Además, posteriormente a la pérdida de dientes pueden sobrevenir deformaciones faciales a causa de la afectación de la cresta residual, deformación labial y pérdida de altura facial, para lo cual es recomendable rehabilitar ya sea con prótesis removible, fija, implantes ${ }^{(9)}$.

Los dientes artificiales son, sin duda, parte del éxito en la confección de prótesis, no solo por la estética, que es de mucha importancia en la actualidad para los pacientes, sino que al mismo tiempo tenemos que valorar la funcionalidad y comportamiento a largo plazo, lo cual nos dará un éxito en el tratamiento ${ }^{(10)}$.

La dureza es una propiedad de los materiales sólidos y se define como la resistencia con la que se oponen estos materiales a ser deformados. La dureza superficial se puede definir como la resistencia de un 
determinado material a ser rayado y a la deformación plástica usualmente por indentación. También puede referirse a resistencia a la abrasión o corte y otro tipo de daños en su superficie(11) (12). El análisis de la microdureza se define como una prueba por medición mediante una indentación, observando la huella que marca el indentador de diamante de forma geométrica contra la superficie de un material a cargas o fuerzas programadas dentro de un rango de 1 a 1000 gramos durante un determinado tiempo.

La microdureza en unidades Knoop y Vickers es obtenida por la medición de la longitud de las diagonales formadas por un indentandor de diamante. Se realiza la medición de la huella con ayuda de un microscopio, el cual nos ayudará a la localización de la indentación, la misma que en la actualidad es medida fácilmente al ya existir los microdurómetros digitales (13). Los microdurómetros son aparatos especializados en la medición de la dureza de diferentes materiales utilizando varios procedimientos llamados ensayos. La dureza de los materiales tiene distintas clasificaciones y para cada una de estas existe un durómetro o microdurómetro ${ }^{(14)}$. El ensayo de dureza de Vickerses una técnica de dureza en la cual un penetrador de diamante muy pequeño y de forma piramidal es forzado hacia la superficie de la muestra con una carga comprendida entre 1 a 1000 gramos durante un determinado tiempo. La marca obtenida es observada al microscopio y esta se mide. El resultado es transformado en un número de dureza. Para esta prueba es muy importante que la superficie a analizar haya sido preparada cuidadosamente para asegurar que la marca sea medida con exactitud. Es una técnica usada para materiales frágiles; en este caso la usaremos en los molares de resina acrílica ${ }^{(15)}$.

La microdureza superficial es una propiedad física que tienen los materiales y se define como la facilidad o dificultad que estos presentan al ser rayados 0 penetrados por un indentador de otro material ${ }^{(16)}$.

Entre las marcas de resina acrílica utilizados en el mercado tenemos:

Dientes artificiales Gnathostar $®$. Están compuestos por: Dimetacrilato 3,3\%-4,0\%, polimetilmetacrilato $95,0 \%-96,0 \%$, pigmentos $0,1 \%-0,4 \%$, iniciadores y estabilizadores $0,5 \%$.

Dientes Duratone-N®. Su composición química es: poli (metacrilato de metilo). Etilenglicol dimetacrilato. Fluorescencia. Pigmentos.
Dientes Trilux®. Compuesto por polimetilmetacrilato, dimetacrilato de etilenoglicol (EDMA) polimerizado, OMC (cerámica orgánicamente modificada), fluorescente y pigmentos biocompatibles.

\section{MATERIALES Y MÉTODOS}

Estudio in vitro. La muestra estuvo conformada por dientes artificiales (molares) fabricados a base de resina acrílica de tres marcas comerciales (Trilux $\AA$, Gnathostar $\AA$ Duratone-n®). Se dividió el estudio en tres grupos de 15 molares para cada marca seleccionada.

\section{Grupo A: TRILUX® \\ Grupo B: Gnathostar® \\ Grupo C: Duratone-n®}

\section{Criterios de inclusión}

Molares 36 y 46 de cada marca seleccionada. Molares que presenten su cara oclusal completamente lisa.

\section{Criterios de exclusión}

Molares que presenten irregularidad en su cara oclusal.

En cada uno de los molares se realizó cortes en sus caras oclusales con la ayuda de motor de alta velocidad Marca WHIP MIX y disco diamantado tratando de obtener una cara lisa. Se realizó moldes de tubo PVC de $3 / 4$ de $1,5 \mathrm{~cm}$ de alto en los cuales se colocó acrílico autopolimerizable en fase plástica y se introdujo cada molar con la cara oclusal hacia la parte superior del molde para su evaluación. Se utilizó acrílico de diferentes colores: rojo para los molares Gnathostar®, azul para los molares Duratone- $n \AA$ y verde para los molares Trilux®.

Una vez terminada la elaboración de las 45 muestras se enumeraron del 1 al 15 los molares de cada marca y se tinturó la superficie a analizar de color azul para facilitar la localización de la indentación. Se procedió con el análisis de microdureza que se realizó en el Laboratorio de Ciencia de Materiales del DECEM, con la prueba de durometría de Vickers a la cual se le programó una fuerza de 200 gramos durante 15 segundos por tres ocasiones para cada molar artificial. Con la ayuda del microscopio incorporado al microdurómetro, se ubicó el área del molar artificial donde fueron realizadas las indentaciones. Luego se realizaron las medidas de sus diagonales gracias al sistema digital de medición otorgado por el fabricante del microdurómetro. Se realizaron tres indentaciones en cada muestra con el fin de obtener 45 valores para cada marca. Con la ayuda de la cámara incorporada en 
el microdurómetro y el software en la computadora se logró capturar el área de la indentación en cada una de las marcas.

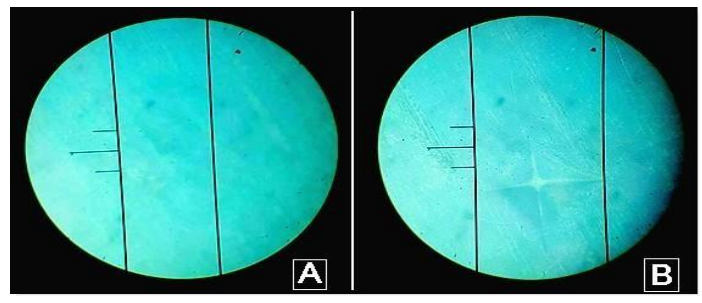

Figura 1. A: Superficie de molar Gnathostar $($ al microscopio 40X sin indentación. B: Superficie de molar Gnathostar ${ }^{\circledR}$ al microscopio 40X con indentación.

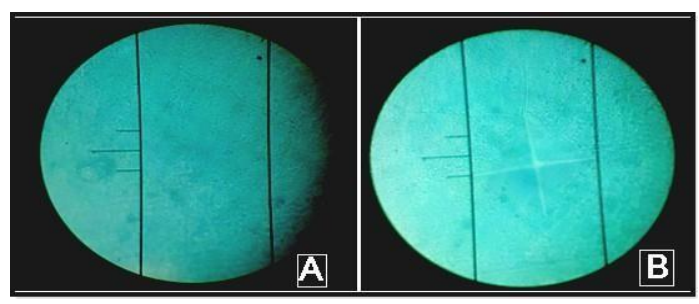

Figura 2. A Superficie de molar Duratonen® al microscopio 40x sin indentación. B: superficie de molar Duratone-n ${ }^{8}$ al microscopio 40X con indentación.

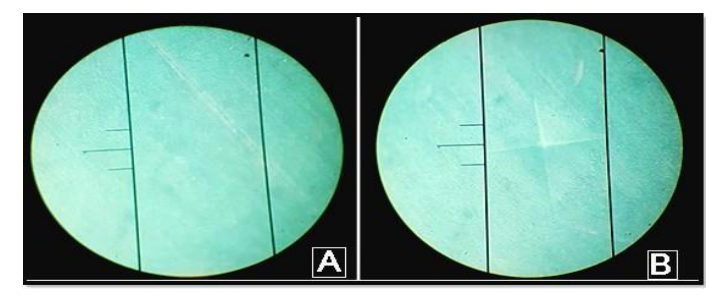

Figura 3. A: Superficie del molar TRILUX® al microscopio $40 \mathrm{X}$ sin indentación. B: Superficie de molar TRILUX® al microscopio 40X con indentación.

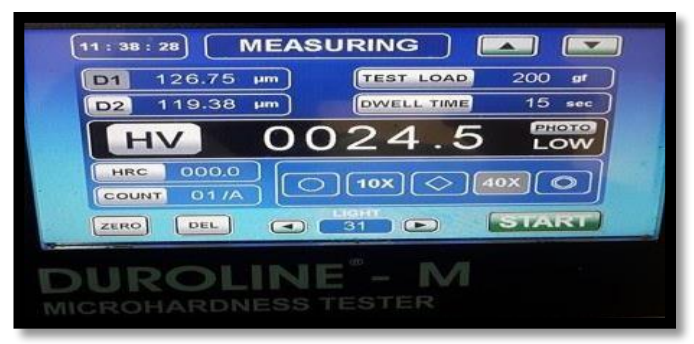

Figura 4. Pantalla digital del microdurómetro con resultado de una indentación.

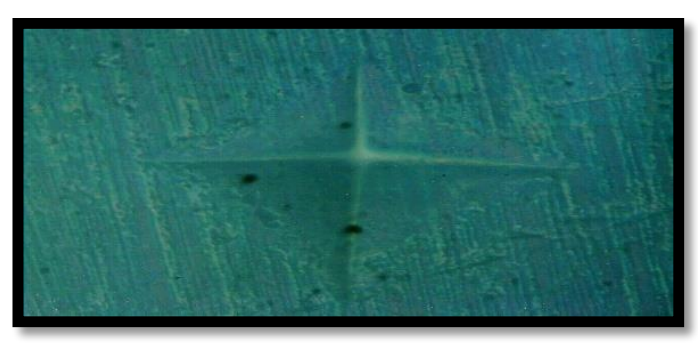

Figura 5. Fotografía con cámara incorporada en el microdurómetro molares Ganathostar®.

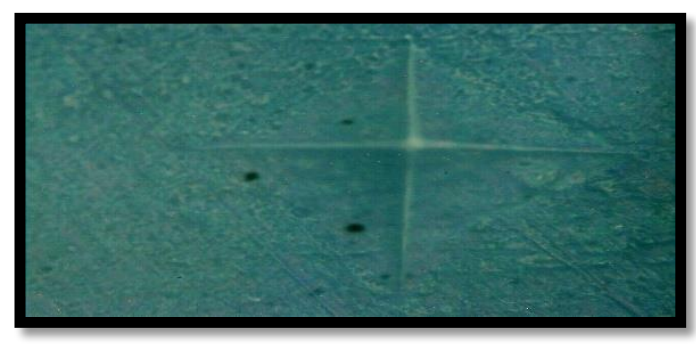

Figura 6. Fotografía con cámara incorporada en el microdurómetro -molares Duratone-n®.

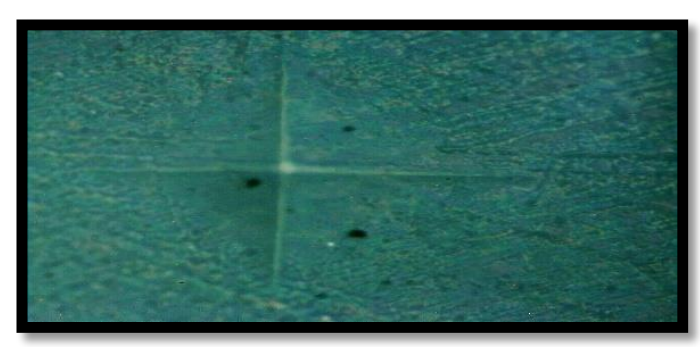

Figura 7. Fotografía con cámara incorporada en el microdurómetro -molares Trilux®. 
La recolección de los resultados se realizó en una tabla previamente diseñada, una para cada marca seleccionada.

\section{RESULTADOS}

El presente estudio evaluó la microdureza superficial de las molares de las marcas Gnathostar ${ }^{\circledR}$, Trilux ${ }^{\circledR}$ y Duratone-n®. Utilizamos 15 molares de cada marca, aplicando la prueba de dureza de Vickers con una fuerza de 200 gramos durante tres ocasiones. Los datos se obtuvieron directamente del software del microdurómetro marca Metkon Duroline-M en unidades de Vickers $(\mathrm{Hv})$ que posteriormente fueron organizados en una tabla previamente diseñada utilizando el Microsoft Word.

Una vez recolectados los datos de microdureza superficial de cada marca seleccionada, se realizó el análisis de los mismos, utilizando el programa de análisis estadístico SPSS statistics 20.0.

Tabla 1. Pruebas no paramétricas: Pruebas de Kolmogorov - Smirnov y Shapiro - Wilk.

\begin{tabular}{lllllll}
\hline \multicolumn{1}{l}{ Pruebas de normalidad } & \multicolumn{5}{l}{ Shapiro-Wilk } \\
& \multicolumn{1}{l}{ Kolmogorov-Smirnov } & \multicolumn{3}{l}{} \\
& Estadístico & gl & Sig. & Estadístico & gl & Sig. \\
\hline GNATHOSTAR &, 141 & 15 &, 200 &, 976 & 15 & 0,933 \\
TRILUX &, 143 & 15 &, 200 &, 941 & 15 & 0,394 \\
DURATONE N &, 154 & 15 &, 200 &, 954 & 15 & 0,583 \\
\hline
\end{tabular}

Fuente: Ing. Jaime Molina

\section{ANOVA: Comparación de medias de la Microdureza Vickers HV.}

Ho: todas las medias son similares.

Ha: Existe una o varias medias que no son similares.

Tabla 2. Análisis descriptivo de los resultados.

\section{Descriptivos}

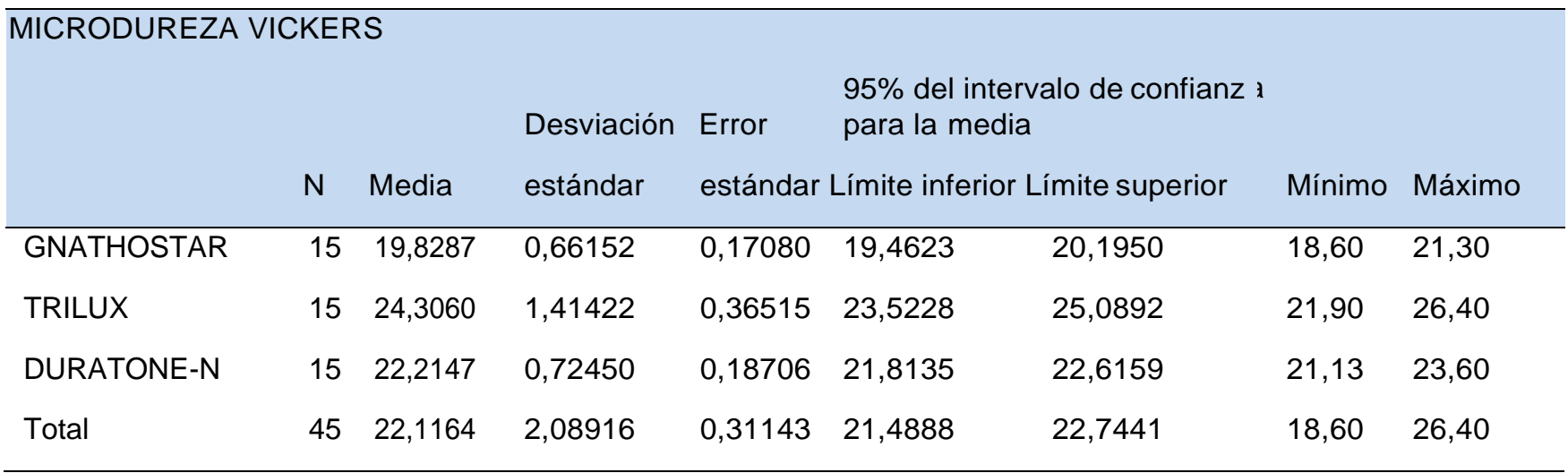




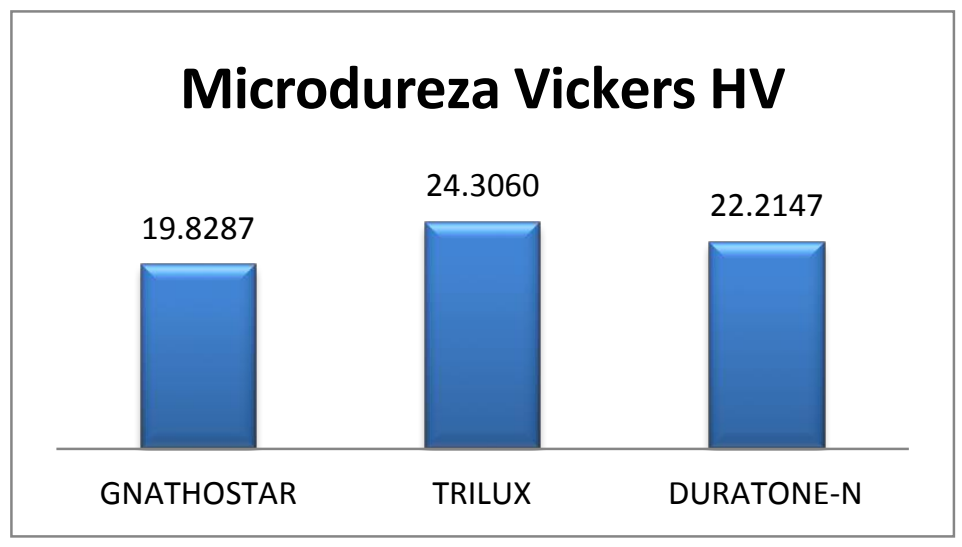

Figura 8. Comparación de medidas de las tres marcas

Fuente: Ing . Jaime Molina

Tabla 3. Microdureza de Vickers.

\begin{tabular}{llllll}
\hline & \multicolumn{5}{c}{ ANOVA } \\
& \multicolumn{5}{c}{ Microdureza Vickers } \\
& Suma de cuadrados & gl & Media cuadrática & F & Sig. \\
\hline Entre grupos & 150,566 & 2 & 75,283 & 76,235 & 0,000 \\
Dentro de grupos & 41,475 & 42 &, 988 & & \\
Total & 192,041 & 44 & & & \\
\hline Prueba de ANOVA & & & & &
\end{tabular}

Tabla 4. Comparaciones Múltiples

\begin{tabular}{|c|c|c|c|c|c|c|}
\hline \multicolumn{7}{|c|}{ Variable dependiente: Microdureza de Vickers } \\
\hline \multicolumn{7}{|l|}{ HSD Tukey } \\
\hline \multirow[b]{2}{*}{ (I) MOLARES } & \multirow[b]{2}{*}{ (J) MOLARES } & \multirow{2}{*}{$\begin{array}{l}\text { Diferencia de } \\
\text { medias (I-J) }\end{array}$} & \multirow[b]{2}{*}{ Error estándar } & \multirow[b]{2}{*}{ Sig. } & \multicolumn{2}{|c|}{$95 \%$ de intervalo de confianza } \\
\hline & & & & & Límite inferior & Límite superior \\
\hline \multirow[t]{2}{*}{ GNATHOSTAR } & TRILUX & $-4,47733$ & ,36286 & 0,000 & $-5,3589$ & $-3,5958$ \\
\hline & DURATONE-N & $-2,38600$ & ,36286 & 0,000 & $-3,2676$ & $-1,5044$ \\
\hline \multirow[t]{2}{*}{ TRILUX } & GNATHOSTAR & 4,47733 & ,36286 & 0,000 & 3,5958 & 5,3589 \\
\hline & DURATONE-N & 2,09133 & ,36286 & 0,000 & 1,2098 & 2,9729 \\
\hline \multirow[t]{2}{*}{ DURATONE-N } & GNATHOSTAR & 2,38600 & ,36286 & 0,000 & 1,5044 & 3,2676 \\
\hline & TRILUX & $-2,09133$ & ,36286 & 0,000 & $-2,9729$ & $-1,2098$ \\
\hline
\end{tabular}

Prueba de Tukey

Fuente: Ing. Jaime Molina 
La prueba dos a dos confirma que las tres medias son totalmente diferentes. El valor más bajo de microdureza es de Gnathostar con una media de 19,8287. Con una microdureza superior se tiene al Duratone-N con una media de 22,2147 y el valor más alto de Trilux con una media de 24,3060.

\section{DISCUSIÓN}

La investigación realizada está dirigida a la elección de dientes artificiales en el tratamiento protésico de pacientes edéntulos parciales y totales. Se valora la microdureza que presentan tres tipos diferentes de molares artificiales con el fin de identificar si existe una diferencia significativa entre los mismos, siendo esta una propiedad muy importante de los dientes artificiales. La microdureza superficial de los molares artificiales promueve la resistencia al desgaste teniendo en cuenta que a mayor microdureza habrá mayor resistencia al desgaste lo que favorece la estabilidad de las relaciones interoclusales evitando la pérdida de dimensión vertical del paciente rehabilitado. Además, se la puede relacionar como indicativo del deterioro de la estructura del molar así como su resistencia al uso durante el acto masticatorio principalmente.

Los dientes artificiales que se emplearon en el estudio están fabricados a base de polimetilmetacrilato, pero en su composición pueden presentar otros componentes como el Etilenglicol dimetacrilato, el cual hace que la estructura de la resina se transforme de lineal a ramificada mejorando las propiedades físicas y químicas en el caso de los molares Trilux ${ }^{\circledR}$ que presentan partículas de cerámica orgánicamente modificada (OMC) ayudando a mejorar la resistencia mecánica, química y a la abrasión.

Assunção, Wirley Gonçalves, Barbosa et al. realizaron un estudio y valoraron el desgaste de ocho marcas de dientes artificiales de resina mediante el método gravímetro y lo analizaron mediante la medición de la diferencia del peso inicial y final tras el experimento. La marca de dientes artificiales Orthosit demostró valores más bajos de desgaste y, por lo tanto, fue la mejor marca de los dientes evaluados para el tratamiento de rehabilitación protésica ${ }^{(17)}$.

Kurser comparó la dureza superficial Knoop de dientes acrílicos fabricados con diferentes tipos de resinas a base de polimetilmetacrilato, con el fin de observar si existen diferencias significativas entre cada uno de ellos y discutir si para mejorar la propiedad de los dientes de resina acrílica interviene el tipo de resina empleada para su fabricación, consiguiendo resultados más semejantes a los de los dientes naturales. Como resultado, se obtuvo que los dientes artificiales fabricados con resinas microrrellenas poseen mayor dureza que los de IPN o resina convencional, pero igual no llegan a tener la misma dureza que los dientes naturales ${ }^{(5)}$.

Mellol, Coppedel, Macedoll et al. estudiaron in vitro la resistencia de desgaste de siete marcas de dientes artificiales (Biolux, Trilux, Dent azul, Biocler, Orthosit, Postaris y Gnathostar) a la abrasión frente a metal (aleación de níquel y cromo) y compuestos antagonistas. Dentro de los resultados se obtuvo que no hubo diferencias estadísticamente significativas entre los diferentes dientes artificiales contra antagonista de los compuestos, pero se logró encontrar diferencias estadísticas al comparar con antagonistas a base de metal. Dentro de las limitaciones de este estudio, se puede concluir que es de mucha importancia la elección del material antagonista al momento de la elaboración de las prótesis ${ }^{(18)}$.

Lugo y Barcelo analizaron y compararon la resistencia al desgaste de tres marcas de dientes artificiales Orthosit (composite), BioTone, (IPN) y Newtek (resina acrílica) con el oclusor de masticación dinámica a 5000, 10.00 y 20.000 ciclos, tomando medidas iniciales y finales con el examinador de superficies rugosas, teniendo como resultados que el desgaste en los dientes de resina acrílica fue mayor que los dientes de IPN y estos tuvieron mayor desgaste que los de composite. En conclusión, se determinó que los dientes de composite presentaron mayor desgaste ${ }^{(4)}$.

De acuerdo con los resultados, se evidenció que existe diferencia en cuanto a microdureza superficial entre los tres grupos seleccionados, La prueba estadística dos a dos de Tukey confirmó que las tres medias son totalmente diferentes. El valor más bajo de microdureza es de Gnathostar con una media de 19,8287 Hv. Con una microdureza superior se tiene al Duratone- $\mathrm{N}$ con una media de $22,2147 \mathrm{Hv}$ y el valor más alto de Trilux, con una media de $24,3060 \mathrm{Hv}$.

Se concluyó que los molares artificiales de la marca Trilux® presentaron mayor microdureza superficial en comparación a los molares de resina acrílica Gnathostar® y Duratone-n®. 
Participación de autoría: PCG,ACG y VSC diseñaron y ejecutaron el estudio y analizaron los resultados y aprobaron la versión final del manuscrito.

Conflicto de interés. Los autores declararon no tener conflictos de interés

Fuente de financiamiento: Autofinanciado.

\section{REFERENCIAS BIBLIOGRÁFICAS}

1. Hechavarria B, Venzant Sucel, Carbonell M, Carbonell C. Salud bucal en la adolescencia. MEDISAN [Internet]. 2013 Ene [citado 2018 Abr 05]; 17(1): 117-125. Disponible http://scielo.sld.cu/scielo.php?script=sci arttext\&pid=S1 029-30192013000100015\&lng=es.

2. Freitas AC. El sistema masticatorio y las alteraciones funcionales consecuentes a la perdida dentaria. Acta Odontol Venezolana. 2008; 46(3): 2.

3. Minaya-Sánchez M, Medina-Solís CE, CasanovaRosado JF, Casanova-Rosado AJ. Peridida de dientes y variables del estado periodontal asociadas en hombres policias adultos. Gaceta Médica de México. 2010;(4): 264-268.

4. Lugo Ancona P, Barceló Santana F. Resistencia al desgaste de dientes artificiales. Revista Odontológica Latinoamericana. 2009; 1(1): 2-4.

5. Kurzer M. Estudio comparativo de dureza en dientes artificiales fabricados con diferentes tipos de resinas acrílicas. Revista EIA. 2006 julio;(6): 122.

6. Lamas C, Paz J, Paredes G, Angulo G, Cardonoso S. Rehabilitación Integral en Odontologia. Odontol. Sanmarquina. 2012;15(1): 21-34.

7. Lucas LVM; Gennari FH; Goiato Marcello Cohello; Dos Santos DM; Moreno A; Falcón-Antenucci RM. Estética en prótesis removibles. Revista Cubana de Estomatología. 2010 junio; 47(2): 224-233.

8. El desafío de las enfermedades bucodentales - Una llamada a la acción global. Atlas de Salud. Federación Dental Internacional. 2016; 1(1): 14.

9. Carr AB, McGivney GP, Brown DT. Prótesis parcial removible. 11 ${ }^{\text {a }}$. ed. Madrid: Elsevier; 2006.
10. Coto S, Martinez A, Rio. JD. Dientes artificiales de composite nanohíbrido: ¿una alternativa a los dientes convencionales? Rev.Gaceta Dental [Internet]. 2011 Septiembre 16; [citado $2018 \mathrm{Abr}$ 05]. En: https://www.gacetadental.com/2011/09/dientesartificiales-de-composite-nanohbrido-una-alternativa-alos-dientes-convencionales-25577/

11. Mendoza O. Evaluación de métodos para la medición de dureza en materiales cerámicos y refractarios de ingeniería. 2004 Mayo; 1(1): 16.

12. Santis A. Análisis de Fallos en Sistemas Aeronáuticos. Madrid: Ed. Paraninfo; 2015.

13. Narváez A. Microdureza de tres tipos de composite fotopolimerizados por luz halógena y por diodoemisión de luz. Granada: Universidad de Granada; 2007.

14. IMOCOM. Durómetros-y-microdurómetros [Internet] 2014 [citado 2017 Marzo 14]. Disponible en: http://www.imocom.com/control-de-calidad/ensayosmecanicos/durometros-y-microdurometros/durometrosy-microdurometros.

15. Callister W. Ciencia e ingeniería de los materiales $7^{a}$. ed. México: Cengage Learning; 2007.

16. Correa E. Microdureza superficial del esmalte dentario ante el efecto erosivo de tres bebidas gasificadas no alcohólicas. Estudio in vitro. Kiru. 2011; 8(2): 88-96.

17. Assunção WG, Barbosa ddb, Goiato MC. Evaluation of the wear rates of eight different brands of artificial resin teeth. Cienc Odontol Bras. 2005; 8(4): 6- 12.

18. Carbone P, Ricciardi A, Macedo A, Chiarello de Mattos M, Silveira R, Faria R. Abrasion wear resistance of different artificial teeth opposed to metal and composite antagonists. Journal of Applied Oral Science. 2009; 17(5): 2-6. 